\section{CHEMIN DE FER LIVERPOOL-SOUTHPORT}

\section{REMPLACEMENT DE LA TRACTION A VAPEUR PAR LA TRACTION ÉLECTRIQUE}

Depuis quelques années les tramways suburbains font aux compagnies de chemins de fer une concurrence redoutable. En effet, les tarits des tramways sont généralement plus bas, leurs frais généraux étant moins élevés, puisque, la circulation se faisant sur route, il n'y a pas eu de terrain à acheter; de plus leurs départs se suivent à de très courts intervalles et leurs arrêts en cour's de route sont plus nombreux. Pour des distances peu considérables, ne demandant qu'un temps relativement court, les hommes d'affaires préférent les tramıays qui, pai leurs départs répétés, leur permettent de mieux utıliser leur temps.

Afin de regagner les voyageurs que les tramways leur enlèvent, les compagnies de chemins de fer doivent donc s'attacher à réorganiser leurs services de manière à ré- les cylindres des machines à vapeur est transformée en énergie électrique qui, à son tour, est retransformée dans les moteur's électriques en énergie mécanique agissant sur les roues motrices. Cette augmentation du nombre des transformations de l'énergie é du nombre des appareils transformateurs entraine inévitablement une diminution de rendement pour des unités de même puissance, et c'est là la cause de l'écliec de la locomotive Heilmann qui opérait toutes ces transformations sur la locomotive ellemême. Mais la traction électrique, tclle qu'on la comprend aujourd'hui, permel de grouper toutes les petiles chaudières et toutes les petites machines à vapeur à échappement à air libre des diverses locomotives, de les réunir en de puissantes unités marchant à condensation ct dont la surveillance et l'entretien sont forcément meilleurs parce qu'elles sont fixes. Il en résulte une augmentation sensible du rendement des machines; par suite, la diminution précédente du rendement provenant de l'augmen-

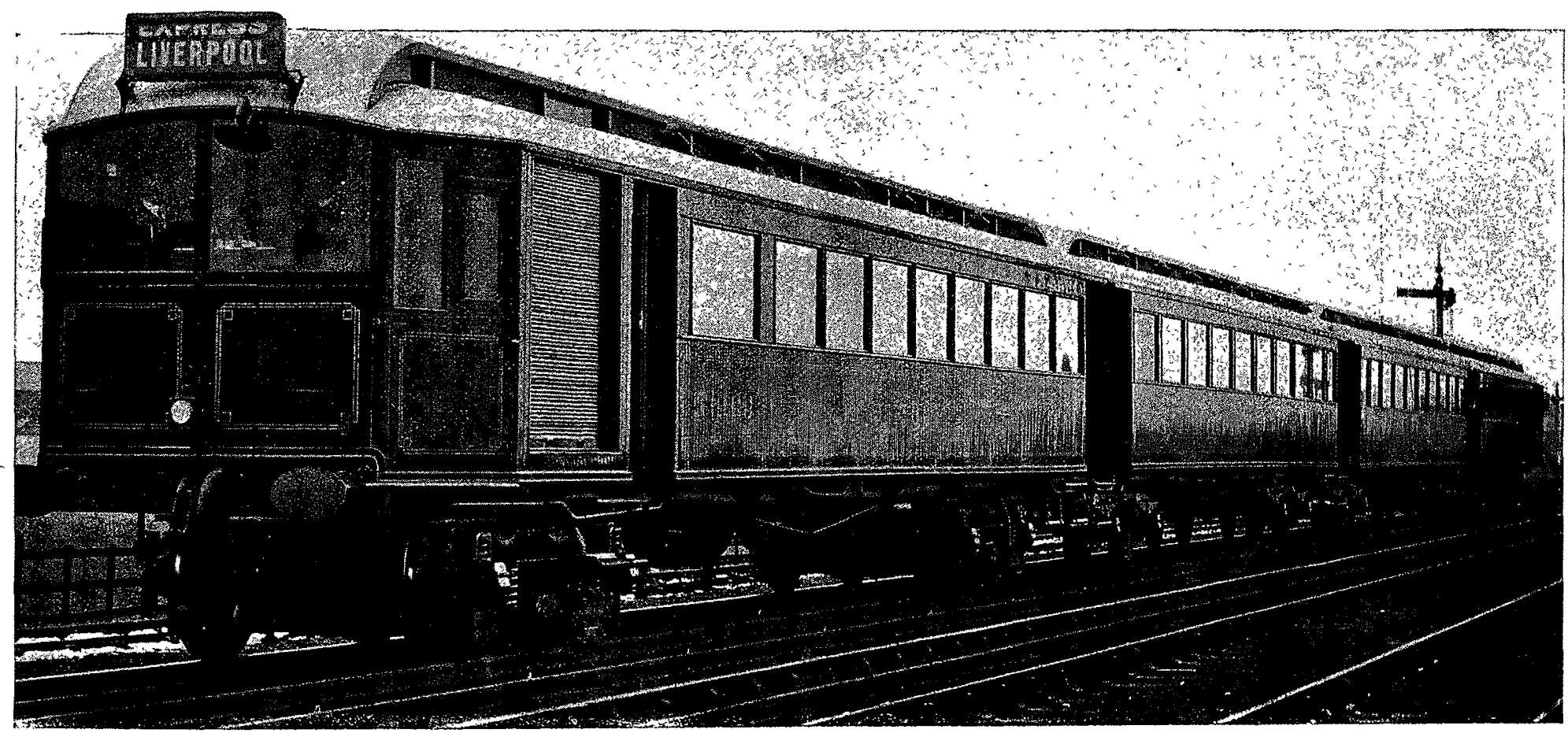

Fig. I. - VUE d'UN TRAIN COMPLET.

duire l'espace de temps entre chaque train, en augmentant le nombre de ceux-ci. L'étude économique de cette question conduit à lemploi de voitures automotrices fréquemment renouvelées avec augmentation de la vitesse cies démarrages et des arrêts.

Il est de toute évidence que l'électricité permet de réaliser facilement ces dernières conditions. Alors l'avantage revient au chemin de fer qui, tout en offrant autant de facilités de départ, permet d'aller plus vite. De plus, le confort est incomparablement meilleur sur un chemin de ler que sur un tramway. 11 en résulte pour la population des villes une plus grande facilité à aller s'établir à la campagne où elle trouve toujour's un meilleur air et souvent les moyens de vivre à meilleur compte.

Avec les locomotives à vapeur, l'énergie calorifique à l'état latent du charbon est transformée dans les chaudières en énergie calorifique utilisable, puis dans les cylindres à vapeur en énergie mécanique agissant sur les roues et engendrant l'effort de traction qui fait progresser le train. Il y a donc deux transformations physico-chimiques s'opérant dans deux appareils distinuts. Avec la traction électrique, le nombre de ces transformations est augmenté; en effet, l'énergie mécanique développée dans tation du nombre des transformations de l'energie se trouve compensée.

Il faut, en outre, tenir compte que les locomotives a vapeur sont obligées de transporter avec elles leur eau ct leur charbon, ce qui constitue un poids mort appréciable et par suite un effort de traction, c'est-à-dire une consommation supplémentaire d'énergie, qui vient contrebalancer la perte en ligne inévitable du transport de l'électricité.

Les machines à vapeur des locomotives étant remplacées par des moteurs électriques, petits par rapport aux grosses unités de la station centrale, il pout sembler quil reste encore une infériorité du rendement dans le cas de la traction électrique; mais le rendement des moteurs électriques est, toutes choses égales d'ailleur's, supéricur à celui des moteurs a vapeur ; les frottements de la machine et des transmissions entre cette machine et les roues sont moins grands dans le cas de l'électricité; enfin les mouvements sont plus doux et moins fatigants pour le materiel et pour la voie, ce qui se traduit en fin de compte par une économie d'argent tout comme une économie de charbon.

Enfin, ct surtout lorsque la traction électrique se fait au moyen de voitures automotrices où il y a un seul mécanicien, on fait une économie de personnel en supprimant le chauffeur. 
C'est dans cet ordre d'idées que M. J.-A.-F. Aspinall, le directeur général de la Lancashire and Yorkshire Company, étudia les moyens de faciliter les communications entre Liverpool et Southport, lieu de plaisance très fréquenté et agréablement situé à l'embouchure de la Ribble River. Un très grand nombre d'hommes d'affaires y habitent toute l'année ainsi que dans les diverses stations qui se trouvent tout le long de la cóte, aussi y a-t-il tous les jours un mouvement très intense de voyageurs allant à Liverpool le matin et en revenant le soir. De plus il y a encore dans la journée un assez crrand mouvement de voyageurs. L'électrification de cette ligne poussée jusqu'à Crossens, à quelques milles plus au nord que Southport, a permis à cette région de rivaliser avec la presqu'ile très fréquentée de Wirral qui se trouve plus au sud.

L'exésution de la transformation de la traction à vapeur en traction électrique fut confiée à la maison Dick Kerr and $\mathrm{C}^{\circ} \mathrm{Ltd}$, dont le siège social est à Londres, comme entrepreneurs généraux. Cette maison possède des usines à Preston, à Lancs et à Kilmarnock. Toutefois le matériel roulant, en dehors des moteurs électriques, fut construit dans les ateliers de Horwich and Newton Works qui appartiennent à la Compagnie du Lancashire and Yorkshire Railway. La transformation fut exécutée en une année, sans entraver en rien l'exploitation à vapeur, et le $1^{\text {er }}$ mars 1904 un train complet circulait par les nouveaux procédés.

La distance entre Liverpool et Southport est d'environ 30 kilomètres (18 milles $1 / 2$ ) et la longueur totale de simplé voie équipée est, en y comprenant le tronçon Southportrrossens, de 75,6 kilomètres (47 milles). La substitution de la traction électrique à la traction à vapeur, en y comprenant le nouveau inatériel roulant, a coûté 340000 livres sterling, soit environ 8 millions et demi de francs, et pourtant cette dépense correspond à la meilleure solution du problème.

La ligne comporte peu de courbes et encore sont-elles à grands rayons; la plus faible courbure se trouve près de Southport et son rayon est de 140 mètres. A quelques exceptions près, la ligne est généralement en pálier; la plus grande pente n'est que de 1,18 pour 100 et encore sur une faible longueur. Entre Liverpool et Southport il y a quatorze stations intermédiaires distantes d'un mille en moyenne; près de Liverpool cette distance est plus faible, près de Southport elle est au contraire un peu plus grande. Entre Southport et Crossens il y a trois stations intermédiaires.

Avec la traction à vapeur il y avait, entre Liverpool et Southport, trente-six trains par jour dans chaque sens. La majorité de ces trains s'arrêtaient à chaque station et ils mettaient 54 minutes entre stations extrêmes. Les quelques trains express, du matin et du soir, à l'usage des hommes d'affaires, meltaient 25 minutes d'un terminus à l'autre. Il y avait en plus, dans chaque sens, trente-huit trains entre Liverpool et Hall Road (11 kilomètres, 25 minutes). Le rapport du nombre des trains dans chaque sens (36) au nombre de milles représentant la distance entre Liverpool et Southport (18 1/2) était de 1,9.

Avec la traction électrique ce rapport est monté à 3,2 . Le nombre des trains entre Liverpool et Southport a été porté de 36 à 65 , et entre Liverpool et Hall Road de 38 à 54 . De plus, le temps du trajet, qui était de 54 minutes pour les trains omnibus entre Liverpool et Southport, est tombé à 37; entre Liverpool el Hall Road il a passé de 25 minutes $\dot{a} 17$. Les trains directs mettent, comme avec la vapeur, 25 minutes pour aller de Liverpool à Southport, seulement en plus des trains du matin et du soir il y en a au moins un par heure dans le courant de la journée, de manière à porter leur nombre à dix-sept. Enfin ces trains express vont jusqu'à Crossens en desservant les trois stations intermédiaires.
La vitesse commerciale des trains express est restéc dans les deux cas égale à 72 kilomètres à l'heure. Celle des trains omnibus est montée de 33,3 kilomètres à 49 .

Cette disposition ne correspond nullement à la limite supérieure des capacites de la ligne, et il n'y aura aucune difficulté à créer un service encore plus actif si le besoin s'en fait sentir.

Afin d'accélérer les mouvements d'entrée et de sortie des wagons, ainsi que cela se fait pour les métropolitains de Paris et de Londres, les voyageurs entrent par une extrémité du wagon el sortent par l'autre.

Ultérieurement, la Compagnie créera un service complet de trains spécialement destinés au transport lapide des malchandises. Dans ce but, elle a dès maintenant entrepris la construction dun certain nombre de fourgons spécialement aménagés pour la manutention rapide des colis, bagages ou marchandises de toute sorte; ces voitures feront plusieurs fois par jour la navette entre Liverpool et Southport de manière à contribuer le plus possible à la commodité et à la rapidité de la déliviance des colis voyageant en grande vitesse.

\section{DESCRIPTION GÉNÉRALE DE L'INSTALLATION}

Afin de réduire la perte en ligne au minimum, l'on a transmis l'énergie sous forme de courants alternatifs triphasés à haute tension. Le courant élechrique est produit directement par les alternateurs de la station génératrice sous la tension de 7500 volts et transmis dans des sousstations ou le voltage est d'abord abaissé par des transformateur's statiques, puis ce courant alternatif est ensuite transformé en courant continu à 650 volts par des commutatrices. Le voltage normal, aux bornes des contrôleurs des automotrices, est de 600 volts

La station centrale à vapeur génératrice d'électricité, de 12000 chevaux indiqués, est située à Formby, sensi. blement au milieu de la ligne et sur les bords de l'Alt River. Les eaux de la rivière servent à l'alimentation des chaudières et à la condensation de la vapeur d'échap. pement.

Il y a quatre sous-stations de transiormation. L'une est située dans l'usine génératrice même; les trois autrès sont situées à proximité de la voie, à Sandhill, Seaforth et Birlidale, et elles sont alimentées chacune par trois cábles à trois conducteurs éprouvés à 30000 volts. Les distances des sous-stations à Liverpool sont respectivement :

De Liverpool

A la sous-station de Sandhill ...........

A la sous-station de Seatorth...........

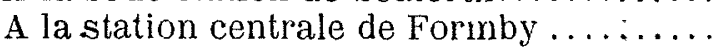

A la sous-station de Birkdale..........

Les deux sous-stations extrêmes fournissent à elles seules le courant nécessaire aux derniers tronçons; chacune d'elles, avec la station intermédiaire voisine, fournit une moitié de l'énergie nécessaire au mouvement des trains entre ces deux sous-stations. Les deux sous-stations de Sandhill et de Seaforth ont un service particulièrement chargé à cause du nombre considérable des trains (238) à destination de Southport ou Hall Road; c'est ce qui explique leur faible distance de Liverpool.

Les choses sont disposées de telle sorte que chaque sous-station peut être déconnectée de la ligne en cas de nécessité.

L'une des caractéristiques de cette ligne est l'emploi d'un troisième rail pour l'amenée du courant et d'un quatrième rail servant de retour, en parallèle avec les rails de roulement.

\section{STATION CENTRALE GENÉRATRICE}

L'architecture de la station centrale est aussi simple que possible et toute décoration inutile a été impitoyablement 
rejetée, par contre l'équipement mécanique et électrique a été aussi soigné que possible et répond parfaitement au but que l'on s'était proposé.

L'usine génératrice comporte deux grandes salles distinctes : la salle des machines de 85 mètres de long sur 20 mètres de large, et la salle des chaudières de 85 mètres de longueur sur 15 mètres de largeur. La toiture de l'usine est metallique et comporte deux travées reposant sur des colonnes également métalliques. Les mul's proprement dits sont en briques. La salle des machines est munie de plusieurs ponts roulants électriques d'une puissance de 20 tomnes.

L'installation comporte quatre machines à vapeur principales d'une puissance de 2310 chevaux chacune, et une cinquieme machine accessoire de 1180 chevaux. Les quatre grandes machines, dont trois d'entre elles peuvent fournir tout lo courant nécessaire à la ligne, sont du type hori- diamètre de $2^{\text {n }} 60$. Elles ont été éprouvées à une pression de $18 \mathrm{~h} 30$. Les épaisseurs des tôles sont de $20 \mathrm{~m} / \mathrm{m} 6$ pour l'enveloppe, 14"' / 3 pour les foyers intéricurs et $19 \mathrm{mms}$ pour les fonds. Elles sont munies de surchauffeurs et d'économiccurs ; ces derniers sont divisés en deux batteries de 720 tubes situées à chacune des extrémités de l'usine. La combustion se fait par tirage forcé obtenu au moyen de deux ventilateurs pouvant dẻbiter un cube d'air sulfisant pour bruler 4,5 tonnes de combustible à l'heure. Ces ventilateurs tournent à raison de 175 tours à la ininute; ils sont aspirants et sont placés entre les économiseurs et la cheminée inonumentale de l'usine, haute de 18 mètres.

Les alternateurs triphasés ont été construits dans les ateliers de MM. Dick Kerr el $\mathrm{C}^{\circ}$, a Preston. Linducteur mobile de chacun des quatre grands groupes électrogènes comporte 40 pôles qui, tomrnant à raison de 75 tour's par

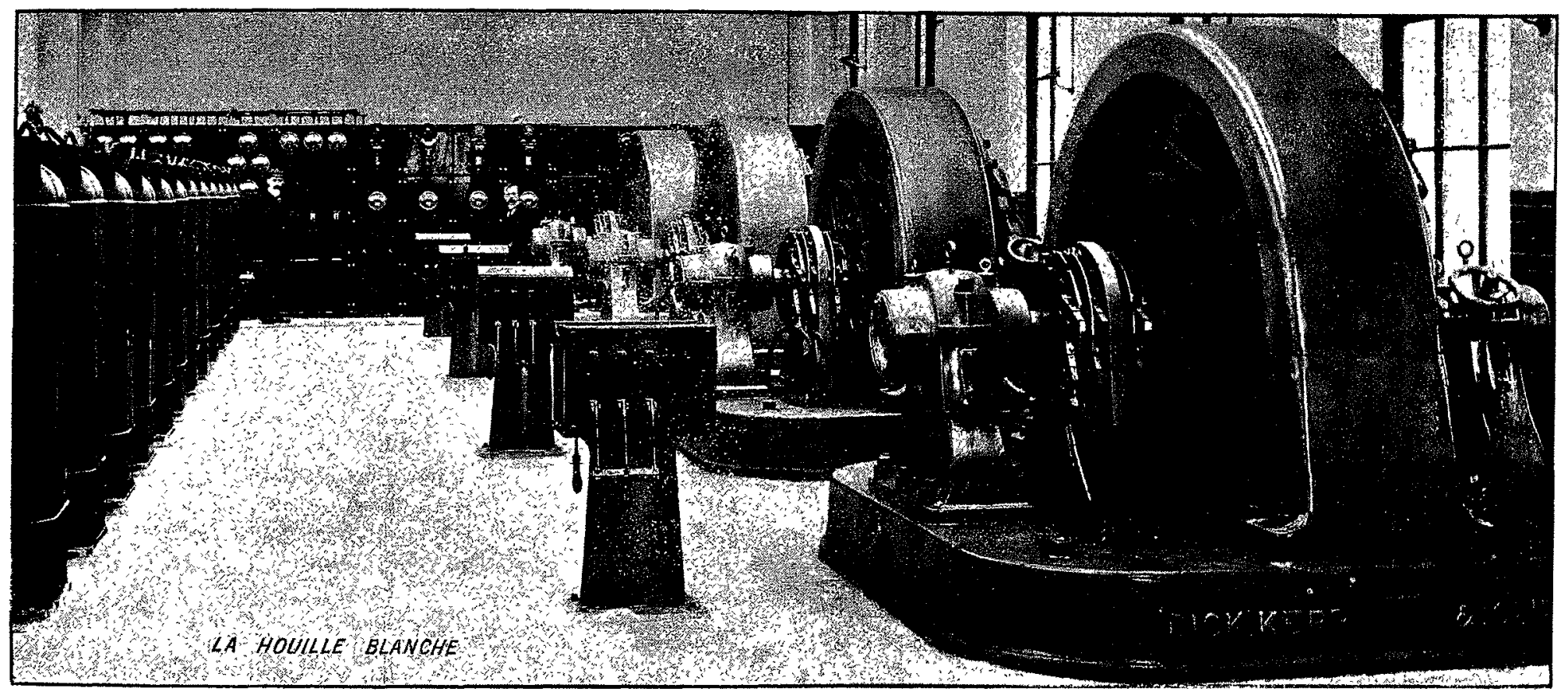

Fig. 2. - Intérielir D'UNE sous-Station de transformation.

zontal Cross Compound à deux cylindres et à condensation; elles tournent à 75 tours par minute et sont directement accouplées à l'inducleur d'un alternateur triphasé de 1500 kilowatts; elles peuvent fournir une surcharge de 20 pour 100. La cinquième machine, qui est verticale, tourne à raison de 94 tours par minute et commande directement un alternateur de 750 kilowatts. Toutes ces machines, ainsi que leurs chaudières, ont été construites par MM. Yates et Thom pour le compte de MM. Dick Kerr et $\mathrm{C}^{\circ}$. La régulation des machines à vapeur a été l'objel d'un soin tout particulier. La distribution de vapeur est complètement coupée si la vitesse de rotation dépasse de 10 poul 100 la vitesse normale. Chacune des grandes machines à vapeur est munie d'un énorme volant de $6^{\mathrm{m}} 70$ de diamètre, indépendant de l'inducteur mobile de l'alternateur correspondant, mais cependant relié solidement avec lui au moyen d'un manchon rigide spécial boulonné sur chacun des bras de cet inducteur.

Les caractéristiques des grosses machines à vapeur sont : diamètre du cylindre $\mathrm{HP}, 0^{\mathrm{m}} 813$; cylindre BP, $1^{\mathrm{m}} 625$; course du piston, $1 \mathrm{~m} 37$. Celles de la petite machine sont: diamètre du cylindre $\mathrm{HP}, \mathrm{O}^{\mathrm{m}} 584$; cylindre $\mathrm{BH}^{\prime}, 1^{\mathrm{m}} 168$; course du piston, 1 m 066 . La pression de la vapeur à l'admission est de $11^{\mathrm{k}} 25$ par centimètre carré.

Les chaudières, au nombre de scize, sont du type Lancashire à double foyer; leur longueur est de $9 \mathrm{~m} 75$ et leur minute, donnent du courant alternatif à 25 périodes pal. seconde. Ces pôles, en acier coulé, sont portés par une couronne en fonte à laquelle ils sont solidement boulonnés. Cette couronne est en deux pièces qui sonl réunies au moyeu par de forts boulons et à la périphérie par de solides clés. Les pièces polaires sont en tôles d'acior doux pour Eviter les couranis de Foucault, elles comportent un vide central pour la ventilation. L'enroulement inducteur est formé de barres de cuivre et sa surface extérieure est $\dot{a}$ nu afin d'augmenter le rolroidissoment. Le poids total de cet inducteur est de 22 tonnes. L'acier des pòles y entre pour $5800 \mathrm{kgs}$ et le bobinage pour $2900 \mathrm{kgs}$.

L'induit qui est fixe est bobiné en étoile avec point cen. tral à la terre. Chaque phase a été essayée à 15000 volts. Le bobinage est logé dans les tôles de l'induit, à raison de deux rainures par phase et par póle. Ces tóles ont été découpées en arc de cercle à l'emporte-pièce avec leur. papier isolant et disposées a joints rompus pour égaliser la réluctance du circuit magnétique, puis fortement comprimées et maintenues par des boulons à l'intérieur d'une flasque circulaire en fonte. Des ouvertures ont été specialement ménagées pour la ventilation. Le poids lotal du stator est de $34400 \mathrm{kgs}$.

La construction de l'alternateur de $750 \mathrm{kws}$ est en tout point semblable à celle des génératrices de $1500 \mathrm{kws}$.

Le courant d'excitation a 125 volts est fourni par trois 
dynamos tétrapolaires de $100 \mathrm{kws}$ qui sont actionnées par des machines à vapeur Williams et Robinsons à grande vitesse (380 tours par minute). Elles servent aussi à l'éclairage de la station et fournissent le courant nécessaire à la commande des ponts roulants, des ventilateurs pour le tirage forcé des chaudières et des autres machines de service.

Le tableau général de l'usine est monté dans une galerie située à la partie supérieure de l'usine, au-dessus de la salle où arrivent et d'où partent les fils haute tension. Cette salle et cette galerie sont construites en matériaux incombustibles. Le tableau se compose de 33 panneaux de $0^{\mathrm{m}} 60$ de large avec leurs instruments de mesure et leurs appareils de manœuvres, voltmètres, ampèremètres, appareils de synchronisation, rhéostats, lampes témoins, disjoncteurs, etc. Voici l'ordre dans lequel sont placés ces panneaux : cinq panneaux pour les cinc alternateur's; un panneau vide; un panneau général; six panneaux haute tension pour le départ des feeders haute tension alimentant les sous-stations de Seaforth, Sandhill et Birkdale; trois panneaux pour les excitatrices; un panneau général pour

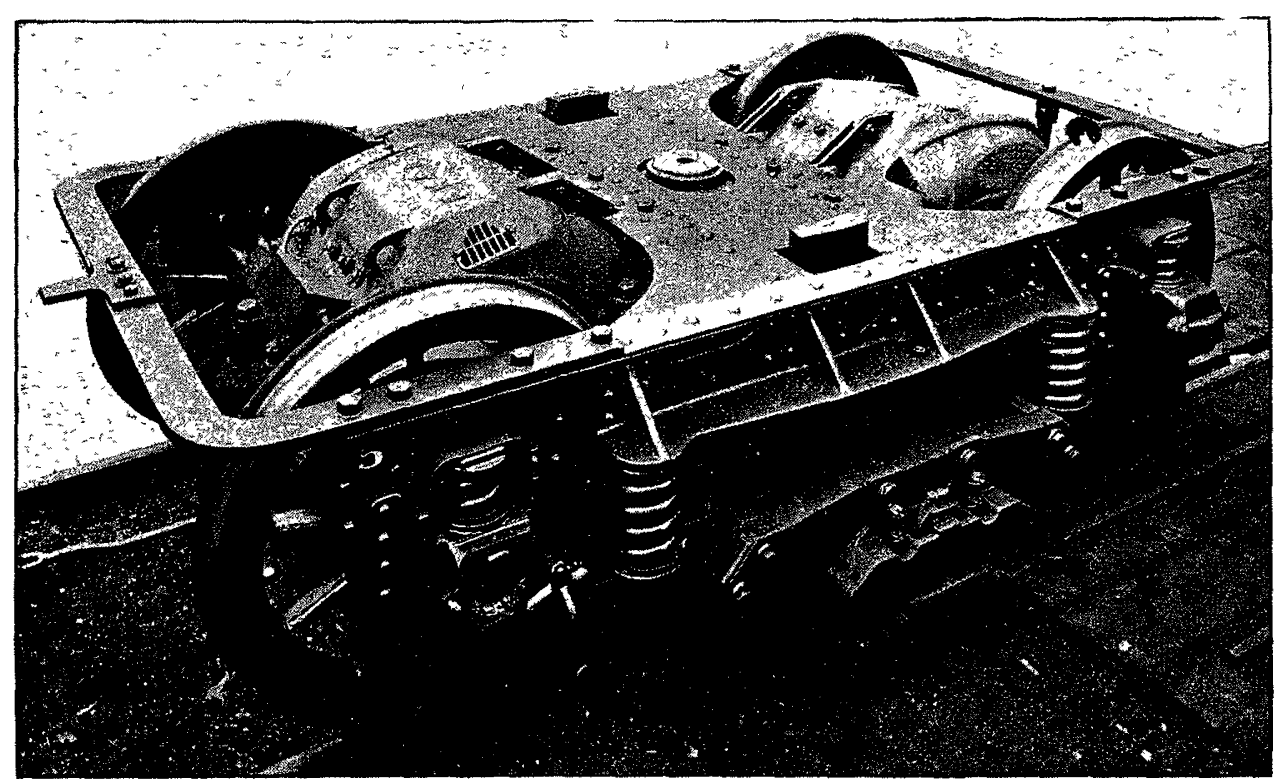

Fig. 3 - Vue d'un bogie moteur.

l'énergie reçue par la sous-station qui se trouve dans l'usine; quatre panneaux commandant les transformateurs statiques de la sous-station ; un panneau vide; quatre panneaux pour les convertisseurs rotatifs; un panneau vide; un panneau général pour l'énergie distribuée par la sousstation; quatre panneaux pour les feeders basse tension alimentant la ligne; enfin un panneau pour l'éclairage et la force motrice de la station centrale. Les panneaux vides ont été prévus pour des attributions ultérieures en prévision d'une augmentation des capacités de l'installation électrique par suite d'une exploitation encore plus intense.

Tout l'ensemble de la haute tension, interrupteurs à huile, transformateurs de voltmètres, shunts dampèremètre, etc., est situé dans la chambre haute tension, de 24 mètres de long sur 3,65 de large, construite en béton armé. En temps normal, personne ne doit pénétrer dans cette chambre, la commande des diverses connexions se faisant du tableau de la galerie supérieure.

Les diverses connexions entre les alternateurs, les panneaux, les transformateurs et les feeders de départ haute tension sont faites soit par des câbles à trois conducteurs, soit par des câbles simples suivant les cas. Ces derniers̀ sont isolés au caoutchouc, les autres sont sous plomb et isolés au papier, tous sont éprouvés à 25000 vults.

\section{SOUS-STATIONS}

Toutes les sous-stations sont identiquement équipées. Celles de Seaforth, Sandhill et Formby sont munies de quatre convertisseurs rotatifs; celle de Birkdale n'en a que trois; toutefois dans chacune de ces sous-stations on a réservé la place nécessaire pour loger une commuta. trice supplémentaire.

Ces convertisseurs sont à huit pôles lamellés et développent 600 kivs sous $650 / 600$ volts, avec une vitesse de rotation de 375 tours par minute. Le courant triphasé arrive par dix-huit frotteurs en cuivre laminé en contact avec six anneaux isolés les uns des autres. Le courant continu sort par huit balais en charbons portés par un collier mobile à volonté au moyen d'un volant de manœuvre. Le poids total de chacune de ces commutatrices est de $20000 \mathrm{kgs}$, dont 10000 pour les électros et 5000 pour l'induit.

Les transformateurs au nombre de trois par commutatrice, un par phase, sont du type à circulation d'air et chaque groupe de trois transformateurs a une capacité de $600 \mathrm{kws}$. L'enroulement basse tension est directement bobiné sur les noyaux; concentriquement et par-dessus se trouve la haute tension. Les tôles du circuit magnétique sont en acier doux recuit et isolées les unes des autres par un papier japon spécial. L'isolement entre le primaire et la terre, ou le secondaire, a été mesuré à 15000 volts; l'isolement entre le secon. daire et la terre l'a été à 2500 volts. Chaque transformateur pèse environ $2700 \mathrm{kgs}$. La circulation de l'air dans les transforma. teurs est assurée pour chaque sous-station par deux ventilateurs électriques de 5 chevaux, capables de débiter 225 mètres cubes d'air à la minute sous la pression de $50 \dot{a}$ 75 mms d'eau.

Chaque sous-station est pourvue d'un tableau de distribution; sur l'un de ses côtés se trouve la basse tension, sur l'autre la haute tension. Ce tableau comprend : un premier tableau général pour l'énergie reçue comportant trois ampèremètres, dont un pour chaque phase, et un wattmètre totalisateur; des panneaux de tranformateurs munis de voltmètres, d'am. pèremètres, d'appareils de synchronisation et d'interrupteur's; des panneaux de com. mutatrices comportant également des voltmètres et ampère. mètres, tant pour le courant principal que pour l'excitation, et des coupe-circuits automatiques; un panneau pour l'énergie distribuée avec ses ampèremètres; quatre panneaux pour les feeders à courant continu avec coupecircuit automatiques; des panneaux pour l'éclairage et la commande des ventilateurs.

Le courant produit par la station centrale génératrice est normalement amené aux sous-stations au moyen de trois câbles. La section du cuivre de ceux-ci est telle qu'en cas d'avarie à l'un d'eux, les deux autres câbles puissenl transmettre toute l'énergie nécessaire sans trop de perte. Ces câbles sont sous plomb, isolés au papier d'une manière spéciale et solidement armés au moyen d'un enroulement en fil d'acier galvanisé de $2 \mathrm{mms}$, afin de les protéger mécaniquement et d'établir un bon contact avec la terré. Chacun des fils de ces câbles se distingue par la couleul diffćrente du papier qui sert à l'isoler. L'épaisseur de l'isolant est de $9 \mathrm{mms}$ entre conducteurs et de $6^{\mathrm{m}} /{ }^{\mathrm{m}} 5$ entre les fils et l'enveloppe de plomb; l'épaisseur de cette del" nière varie de 3 à $\mathrm{mms}$ suivant le diamètre du câble. Tous ces câbles ont été éprouvés, pendant une heure. à 30000 volts, entre fils et entre fils et terre.

Les trois câbles ont été logés dans des caniveaux en bois 
goudronné et créosoté enfouis dans le sol ct recouverts de tuiles et, lorsque les conditions locales ont obligé a les faire passer au niveau du sol, ces caniveaux en bois ont été remplacés par des augets en forte tôle d'acier.

\section{ÉQUIPEMENT ÉLECTRIQUE DE LA VOIE}

Une des particularités de cette ligne est que l'amenéc du courant se fait par un troisième rail et que le retour de co courant a lieu à la fois par les rails de roulement et par un quatrième rail, relié électriquement avec ceux-ci à chaque éclisse. Cette disposition diminue la résistance électrique de l'ensemble du circuit de retour et de plus elle facilite beaucoup un remplacement rapide de rails de roulement, ce qui est particulièrement intéressant sur une ligne à grand trafic.

Le troisième et le quatrième rail, tous deux d'égale section, sont fabriqués avec un acier doux de haute con-
Dans les passages à niveau, le troisième rail est interrompu à une certaine distance de chaque côté et ses extrém mités sont réunies entre elles au moyen d'un cảble souterrain; le quatrième rail est simplement remplacé par un fil nu reposant sur le sol.

La prise de courant se fait au moyen de sabots froltcurs qui appuient sur le troisième rail. Le courant s'en va par les roues, les rails de roulement et le quatrième rail.

\section{MATÉRIEL ROULANT}

Ainsi que cela a lieu sur la plupart des Compagnics anglaises, les trains se composent de voitures de première et de troisième classes sans seconde classe. Chaque train est en général formé de quatre voitures: deux voitures automotrices de troisième classe situécs cn tête et en queue, ct deux voitures de première au milieu. Les automotrices sont actionnées par quatre moteurs de 150 chevaux placés

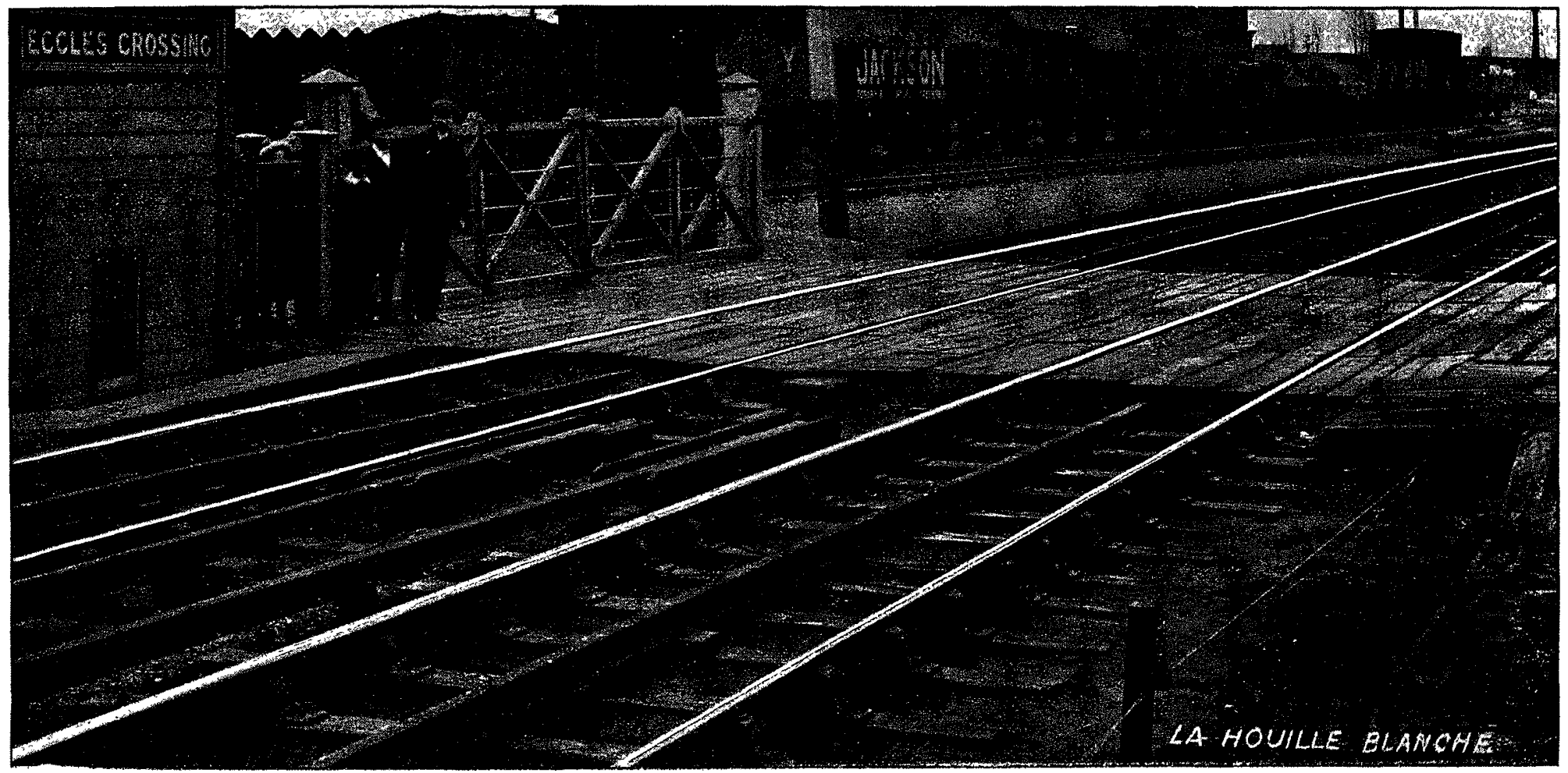

Fig. 4. - Vue d'un passage a niveau montrant la disposition des rall.s conducteurs de courant ET LEUR INTERRUPTION SOUS LE PASSAGE.

ductibilité électrique; leur résistivité, comparée à celle du cuivre, n’a jamais été supérieure à 7,25 fois celle ci et la plupart du temps elle a été trouvée bien inféricure à ce chiffre. Ces rails sont du type Vignole, ils pèsent $44 \mathrm{kgs}$ par mètre courant et leur longueur est de $18^{\mathrm{m}} 30$. Ils sont posés bout à bout et à joints fixes par sections de $91^{\mathrm{m}} 50$, chaque section est reliée à la suivante par un joint mobile pour la dilatation.

Le troisième rail est supporté tous les trois mètres, c'està-dire toutes les trois traverses, par des isolateurs en pâte de granit et maintenu par deux éclisses. Son axe longitudinal est exactement à $1^{\mathrm{m}} \mathrm{32}$ de l'axe de chaque voie de roulement, tantôt à l'intérieur entre les deux voies, lantôt à l'extérieur el il dépasse de $7 / \mathrm{m} 62$ les rails de roulement. Ces dimensions sont celles qui ont été adoptées, pour les grandes lignes de chemins de fer, au Congrès qui s'est tenu à Clearing-House, le 3 mars 1903.

Le quatrième rail est simplement supporté par des blocs de bois nu reposant directement sur les traverses au milieu de la voie. Des fils de cuivre relient, à chaque extrémité, les rails de roulement au quatrième rail de retour. sur les deux bogies, ce qui fait huit moteurs par train. Le courant est amené aux moteurs par un frotteur on acier disposé de chaque côté des bogies moteurs et qui prend contact avec le troisième rail.

Les voitures des deux classes ont $18 \mathrm{~ms}$ de long sur $3 \mathrm{~ms}$ de large. Elles reposent sur deux bogies distants de $12 \mathrm{~ms}$ d'axe en axe et larges de $2^{\prime \prime} 4$. Les roues ont $1 \mathrm{~m} 06$ de diamètre. Ces voitures permettent de transporter 66 voyageur's en promière classic et 69 en troisième, soit 270 voyageur's par train. Les voitures automotrices pèsent 44 tonnes, les autres 26 , de telle sorte que le poids mort total d'un train est de 140 tonnes.

Les automotrices comprennent un grand comparliment de troisième classe pour les voyageur's, un petit compartiment pour les bagages et une cabine pour le mécanicien; celle-ci est toujours en tête du train. L'entrée des voyageurs se fait aux extrémités de chaque voiture au moyen d'un petit vestibule.

Les sièges sont tout d'abord disposes longitudinalement, laissant entre cux un large passage pour l'entrée ou la sortie, puis ils sont ensuite disposés transversalement 
avec un passage longitudinal. Dans les voitures de première classe il y a deux sièges de chaque côté de ce passage longitudinal, tandis que dans les wagons de troisième il y en a trois d'un côté et deux de l'autre. Les voyageurs de première classe ont la faculté d'aller d'un bout à l'autre du train, des soufflets ayant été ménagés entre chaque voiture.

Chaque compartiment est éclairé et chauffé à l'électricité ; des interrupteurs spécialement affectés à cet usage sont placés à l'entrée du vestibule-d'entrée de chaque voiture et peuvent être manœuvrés par une clé que possède le conducteur du train.

Les bogies moteurs sont entièremement en acier. Ils portent de chaque côté un frotteur en acier coulé qui pèse $41 \mathrm{kgs}$ et qui est maintenu on place par une solide pièce
Le sifflet des locomotives à vapeur est remplacé par une corne sonore de construction spéciale en communication avec les réservoirs à vide.

Ainsi que cela a été dit précédemment, un train complet est actionné par huit moteurs dont les axes sont perpendiculaires à celui de la voie et qui actionnent directement les roues des bogies au moyen d'un engrenage à simple réduction dans le rapport de 10 à 6,9. Ces moteurs ont été établis pour pouvoir développer 150 chevaux à la vitesse de 470 tours par minute. Leur poids est de $3000 \mathrm{~kg}$ s dont 900 pour l'induit et 230 pour les engrenages. Ils ont été largement calculés de manière à supporter pendant quelques instants de fortes surcharges sans élévation trop sensible de température. Le circuit magnétique est en acier fondu avec quatre pôles feuilletés, rapportés et soli-

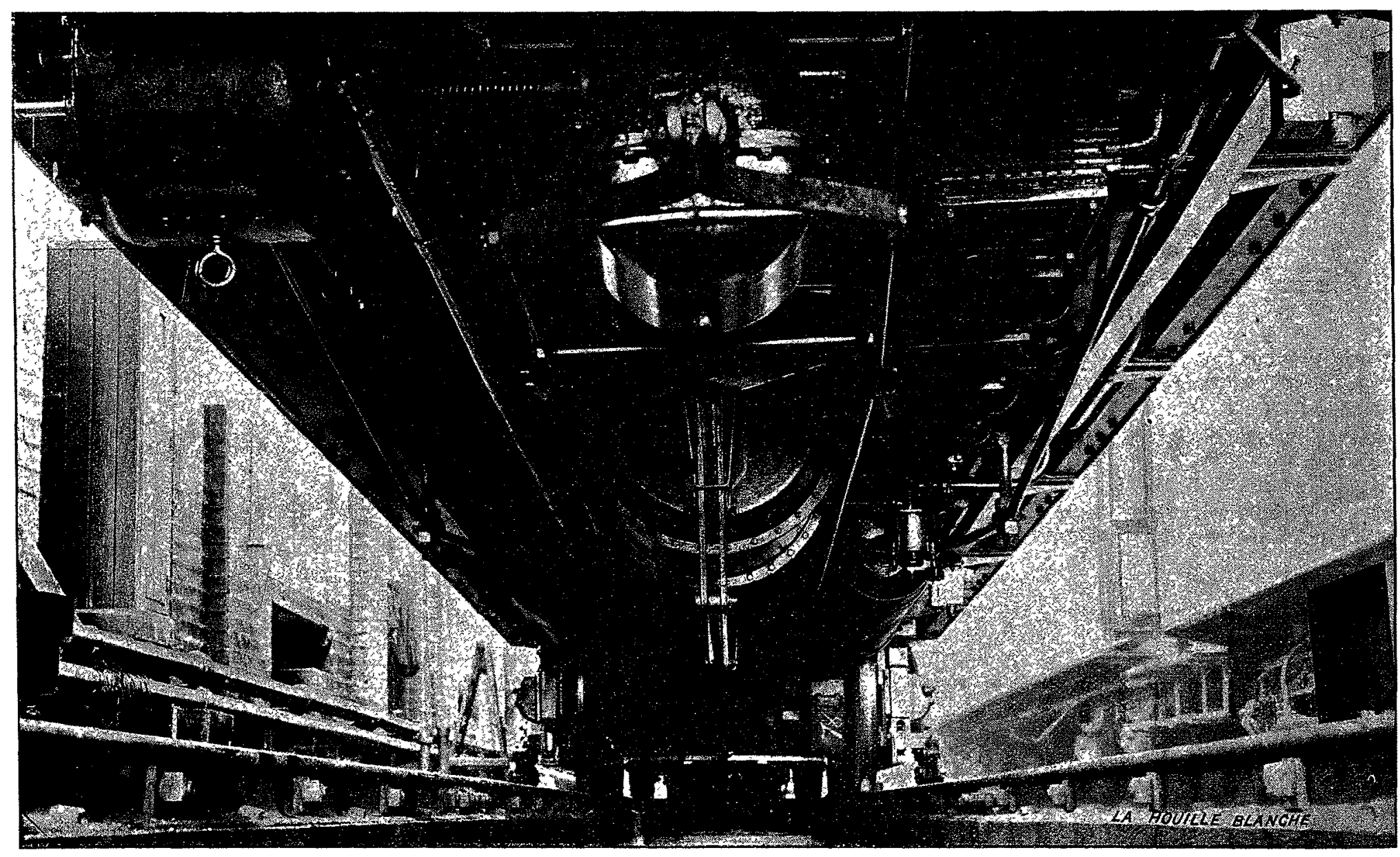

Fig. 5. - Vue par dessous d'Une voiture automotrice montrant la disposition des moteurs, des résistances, ET DE LA COMMANDE DES FREINS.

de bois attachée au cadre du bogie servant à la fois de support et d'isolant. En marche normale, les quatre frotteurs situés d'un même côté du train captent le courant en quatre points différents. Lors des passages à niveau où le troisieme rail est interrompu il y a toujours deux frotteurs, soit de la voiture de tête, soit de la voiture de queue, qui sont en contact permanent. Des frotteurs, le courant se rend au contrôleur à travers un câble flexible.

Le freinage s'obtient au moyen du frein à vide qui est en usage sur les autres locomotives à vapeur de la même Compagnie. La pompe à vapeur est remplacée par une pompe mue par un électromoteur de 3 chevaux sous 600 volts. La commande des valves se fait électriquement et instantanément d'un bout à l'autre du train. Des régulateurs électropneumatiques maintiennent automatiquement un vide constant dans les réservoirs dont chaque voiture est munie. Un manomètre placé dans la cabine du mécanicien lui indique constamment le degré du vide. dement maintenus avec la carcasse magnétique par des boulons. Les tôles d'acier, munies de trous de réfrigération, sont directement calées sur l'arbre. Le collecteur, en cuivre étiré, et ses balais, en charbon, sont facilement accessibles.

L'une des grandes difficultés qui se présentent dans la commande des trains à unités multiples réside dans le contrôleur par lequel il est pratiquement difficile de faire passer tout le courant nécessaire. Le contrôleur Dick Kerr employé ici comporte deux cylindres, chacun d'eux contrôlant une des moitiés du train, l'un l'automotrice de tête, l'autre l'automotrice de queue. Sans rien changel' à l'unité générale du train, celui-ci peut être divisé en deux unités distinctes. La conduite du train est entièremént faite par le mécanicien qui se trouve dans la cabine du devant de la première voiture.

Les deux cylindres du contrôleur sont actionnés par 
une manette et chacun d'eux est muni d'une forte bobine magnétique pour souffler les arcs. On a aussi prévu deux interrupteur's à soufflage magnétique coupant automatiquement le courant en cas de surcharge et réglés respectivement à 500 et 2000 ampères. Le contrôleur comporte aussi une manette de renversement qui, au moyen d'une disposition spéciale, actionne l'inverseur de chacun des huit moteurs.

Les moteurs sont disposés en quatre groupes marchant toujours en parallèle et chaque groúpe comporte deux moteurs, en série aux démarrages, avec un interrupteur automatique coupant le courant amené à ce groupe en cas de trop forte surcharge ou de court circuit. Cet interrupteur est commandé par un électro-aimant disposé sur le circuit de ce groupe. Lorsque le courant est coupé, c'est-àdire que le circuit du groupe correspondant est ouvert, une petite plaque, disposée sur le côté extérieur du bogie, tombe et montre le mot " ouvert » peint en grosses lettres blanches sur un fond écarlate, ce qui attire immédiatement l'attention. Ces interrupteurs peuvent aussi être manœuvrés à la main et isoler le groupe correspondant.

Les deux automotrices de tête et de queue sont commandées à volonté de l'un ou de l'autre bout du train, de telle sorte qu'au terminus le mécanicien n'a qu'à changer de poste pour repartir en sens inverse. Les connexions entre les sabots frotteurs, les contrôleur's et les moteurs se font au moyen de quatre câbles dont l'un est triple et sert pour l'éclairage, le chauffage et l'inverseur des moteurs.

Aux démarrages le courant atteint jusqu'à 2000 ampères, mais il tombe ensuite à $500 / 450$ ampères lorsque la vitesse normale est atteinte, les huil moteurs étant alors en parallèle. Les résistances de réglage sont disposées sous la voiture. En marche normale le contrôleur et les résistances arrières sont hors circuit, mais chaque automotrice est prévue de telle façon que ses résistances permetlent de contrôler tout l'ensemble du train.

La transformation de la traction à vapeur par la traction électrique, sur la ligne Liverpool-Southport, a réalisé toutes les espérances qui avaient conduit à adopter cette solution. Nul doute que cet exemple ne soit suivi dans bien d'autres endroits, autour des grands centres indus. triels ou commerciaux, surtout lorsque ceux-ci peuvent être économiquement alimentés par des transports d'énergie provenant de puissantes usines hydro-électriques comme il s'en trouve déjà dans les Alpes. En cas d'insuffisance d'eau au moment de l'étiage, une partie de l'énergie pourrait être fournie par des machines à vapeur de secours, régularisant ainsi le débit électrique absorbé par les chemins de fer que le débit insuffisant de l'eau ne permettrait pas de fournir.

Une pareille transformation est également intéressante pour les lignes de montagnes traversant des régions pittoresques très fréquentées des touristes. L'emploi d'automotrices électriques permet de supprimer la locomotive à vapeur, qui doit être d'autant plus lourde que la rampe est plus forte et le nombre des royageurs plus considérable, et de diminuer en conséquence le poids mort du train et par suite l'effort de traction à la jante des roues motrices.

Ce sont ces diverses considéralions qui nous ont engagé à essayer de décrire la précédente transformation.

\section{H. BELLET.}

Dans le prochain numéro nous publierons une note de $M$. Ribourt sur des jaugeages effectués, par divers procédés, $\dot{a}$ Brides-les-Bains (Savoie).

\section{INFLUENCE DE L'HYSTÉRÉSIS SUR LA} MARCHE DES ALTERNATEURS EN PARALLÈLE

Communication de M. Boucherot au Congrès de Saint-Louis.

Dans une communication faite il y a quelques mois par M. H.-H. Barnes, à l'American Institute of Electrical Engineers, cet ingénieur a signalé un fait très curieux qu'il a appelé "cumulative surging ) se produisant dans certains cas avec des alternateurs volants couplés en parallèle (I).

On supprime les régulateurs de deux groupes électrogènes en les bloquant pour que l'admission soit constante. On les amène à la même vitesse autant que l'on peut, on couple. Alors les machines, au lieu de se mettre en concordance de phase après quelques oscillations, oscillent au contraire de plus en plus avec leur période propre d'oscillation $T$ et l'amplitude de ces oscillations crôtt assez rapidement jusqu'au décrochage. Ce phénomène ne saurait être attribué a la résonance, car dans les cas spéciaux où il s'est produit, la période propre $T$ était deux à trois fois plus longue que la période de rotation. Il ne saurait être non plus attribué aux régulateurs de vitesse, puisque ceux-ci étaient supprimés. Il semble donc y avoir une cause encore inconnue de perturbations lors de la marche en parallèle de deux alternateurs.

Je crois que l'on peut dès maintenant proposer deux explications de ces faits. Il peut y avoir au moins deux causes de perturbations ; il y en a peut-être encore d'autres.

On trouvera dans une autre communication au Congrès, sur ( La variation cinétique de tension », une première explication que je résumerai ici en quelques mots (2):

Lorsqu'un alternateur a une variation de vitesse périodique, si son excitatrice est conduite par lui-même, soit qu'elle soit placée en bout de l'arbre, soit qu'elle soit conduite par engrenages ou courroie, les variations de tension de l'alternateur sont un peu en retard sur les variations de vitesse. Le décalage $\varphi_{4}$ des variations de tension sur les variations de vitesse qui est toujours compris entre o et $\frac{\pi}{2}$ peut, sans que les circonstances soient très anormales, être voisin de $\frac{\pi}{4}$. Si l'on réunit alors deux tels alternateurs en parallèle, il y a dans chacun, du fait même des oscillations, de quelque nature qu'elles soient, un couple amplificateur, de signe contraire à lamortissement, qui a pour valeur approchée :

$$
\frac{E_{\mathrm{er}} I_{\mathrm{cc}}}{\Omega_{\mathrm{m}}} \frac{\omega \varepsilon}{\alpha} \frac{\Xi_{\xi}}{4} \sin 2 \varphi_{\mathrm{s}} \sin \alpha t
$$

lorsque la vitesse de l'un d'eux est:

$$
\Omega_{2}(1+\varepsilon \sin \alpha t)
$$

$\Omega_{\mathrm{m}}$ vitesse angulaire moyenne, variation relative de cette vitesse, variation relative correspondante de la tension,

(1) Voir La Houille Blanche, aoùt 1904.

(2) Nous donnerons cette étude dans un prochain numéro. 